\title{
Radiación Ultravioleta. Análisis de su Comportamiento Estacional en Diferentes Sectores de la Región Piura. Costa Norte del Perú
}

\author{
Dedios Mimbela Ninell Janett \\ Servicio Nacional de Meteorología e Hidrología. \\ SENAMHI \\ ndedios@senamhi.gob.pe/ndedios@cidmaperu.org
}

(Tipo de Artículo: Investigación Ciencia y Tecnología. Recibido el 16/11/2015. Aprobado el 18/01/2016)

\begin{abstract}
Resumen. Se denomina radiación solar a los conjuntos de radiaciones electromagnéticas emitidas por el sol. Esta energía juega un papel determinante en diversos procesos en la biosfera, su sobre exposición genera impactos sobre la salud de personas, animales y plantas. El presente artículo desarrolla un análisis sobre el comportamiento estacional de la Radiación Ultravioleta en diferentes sectores de la Región Piura. Costa Norte del Perú. La metodología aplicada considera la información procedente del sensor IUV biometer modelo 501-A (Solar Light), SN 10734 (respuesta espectral 250/380 nm), periodo 2008-2011. Desde un aspecto cualitativo, hemos empleado la escala de valores del Índice UV, definida de 1 a 14+ según la Meteorological World Organization incluidas las recomendaciones efectuadas por instituciones dermatológicas. Los resultados, demuestran la variabilidad del índice de radiación ultravioleta en función de la estacionalidad, a su vez que Piura presenta niveles de radiación ultravioleta entre altos a extremos durante todo el año lo cual representa una alerta a fin de informar a la población sobre las formas de cómo evitar los problemas e impactos en la piel.
\end{abstract}

Palabras clave. Radiación Ultravioleta, Monitoreo, Prevención.

\section{Ultraviolet Radiation. Analysis of their seasonal behavior in different sectors of the Piura region. Coastal North of Perú}

\begin{abstract}
Solar radiation to the electromagnetic was emitted by the sun. This energy plays an important role in various processes in the biosphere, its over exposure generates impacts on the health of people, animals and plants. This article develops an analysis of the seasonal behavior of the UV radiation in different sectors of the Piura Region. Coast North of Peru. The methodology was applied to use information from the sensor UVI biometer model 501-A (Solar Light), SN 10734 (spectral response 250/380 nm), period 20082011. From a qualitative aspect, we have used the scale of values of the UV index, defined from 1 to 14 , according to the World Meteorological Organization including the recommendations made by dermatological institutions. The results show the variability of the index of ultraviolet radiation according to the seasonality, turn Piura presents levels of ultraviolet radiation between high ends throughout the year which represents an alert in order to inform the population about the ways of how to avoid problems and impacts on the skin.
\end{abstract}

Keywords. Ultraviolet Radiation Monitoring, Prevention.

\section{Introducción}

La declinación global de la concentración de ozono en la atmósfera, ha sido comprobada con el uso de instrumentos a bordo de satélites artificiales o desde mediciones terrestres. En este sentido, el índice de la radiación ultravioleta (IUV) mide la intensidad de la radiación UV solar en la superficie terrestre. Definido como la porción del espectro electromagnético entre 100 y $400(\mathrm{~nm})$ nanómetros $(\mathrm{nm})$, está formada por tres subtipos distintos ('A','B', y 'C') en función de la banda del espectro que ocupan. En el rango de $280 \mathrm{~nm}$ a $315 \mathrm{~nm}$ la radiación UV-B es más peligrosa que los rayos UVA, siendo implicado como la causa principal de cáncer de piel, quemaduras solares y cataratas oculares [1]. El deterioro progresivo que ha sufrido la capa de ozono en las últimas dos décadas [2, 3, 4], puede tener consecuencias importantes en los seres vivos en la tierra, ante un incremento eventual de la radiación solar ultravioleta B (UVB: $280-320 \mathrm{~nm}$ atenuada por la capa de ozono [3]. Estas radiaciones tienen bastante energía para producir daño y perjuicios en los sistemas biológicos dado que cada especie de ser vivo, incluyendo individuos diferentes dentro de una misma especie, tienen una respuesta diferente a una irradiancia solar ultravioleta(UV) dada,[5, 6].
Tanto las radiaciones UVA como la UVB juegan un papel importante en la patogénesis de enfermedades fotosensibles como la erupción polimórfica por la luz [7, 8], inmunosupresión [8], foto envejecimiento [9] y foto carcinogénesis [10]; [11]. Sin embargo, UVB tiene mil veces mayor capacidad de producir quemadura solar y daño genotóxico [12]. En la actualidad los tumores de piel son el tipo más frecuente de neoplasias humanas [13]. La exposición intensa, prolongada y excesiva al sol puede provocar cáncer de piel, sin importar la pigmentación de esta [14].

En el Perú, según el Instituto Nacional de Enfermedades Neoplásicas (INEN), se diagnostican entre 3000 y 5000 casos de cáncer de piel por año, un gran porcentaje no detectado a tiempo, comprometiendo la vida de aquellos que la padecen [15]. Entre la población vulnerable se destacan a los niños que constituyen el grupo de mayor riesgo, ya que pasan mucho tiempo al aire libre, siendo el tiempo de exposición al sol tres veces mayor que la de los adultos [15]. Asimismo, las personas de edad son más propensas al cáncer de piel como consecuencia de una mayor exposición total a la luz solar [14]. En un estudio con 122 pacientes en la ciudad de Trujillo-Perú, se encontraron que las mujeres eran más susceptibles de 
Ing. USBMed, Vol. 7, No. 1, Enero-Julio 2016

adquirir fotodermatosis idiopática en un $81.73 \%$ en relación a los varones, siendo la edad promedio de 28 años en los varones y 32 años en las mujeres y un tiempo de desarrollo de la enfermedad promedio de 16,12 y 6,78 meses respectivamente [16]. En el caso de los deportistas o personas que realizan sus actividades económicas al aire libre, como agricultores o pescadores forman parte del grupo de riesgo [17]. En relación a su ubicación geográfica, la cercanía hacia el ecuador, permite que más intensas sean las radiaciones ultravioleta y por tanto mayor daño, a su vez a una mayor altitud la intensidad de las radiaciones ultravioleta aumenta 5\% por cada 1000 metros de altitud [18,14]

En cuanto a su medición, los instrumentos ubicados en satélites han alcanzado gran precisión y permitido un muestreo permanente del planeta entero. De estas mediciones se han derivado niveles de irradiación solar UV y dosis eritémicas en toda la tierra a partir de los datos satelitales del instrumento TOMS. El modelamiento teórico a su vez es una herramienta de análisis importante que contribuye conjuntamente con el de los satélites a contrastar los resultados de las mediciones obtenidas desde estaciones ubicadas en tierra y satelitales.

En Perú, el Servicio Nacional de Meteorología e Hidrología (SENAMHI), realiza mediciones del Índice de Radiación Ultravioleta para todo el territorio nacional y que son difundidas a través de su página web y en diferentes medios de comunicación. En este sentido surge la necesidad de despejar algunas incógnitas sobre cuál es el nivel de incidencia del índice de radiación ultravioleta y como es su comportamiento temporal en la región Piura-Perú. Con el propósito de dar respuesta a nuestras interrogantes es por el cual, el presente artículo hace un análisis del monitoreo de la radiación ultravioleta y analiza si esta se encuentra influenciada por los niveles altitudinales (costa y sierra).

\section{Metodología}

\section{1 Área de Trabajo}

La región Piura está ubicada en el extremo noroccidental del Perú. Con una superficie de 35,892.49 km2 y una población de 1'676,315 habitantes [19], es el segundo departamento más poblado del Perú. Sus coordenadas geográficas se encuentran entre los $4^{\circ} 04^{\prime} 50^{\prime \prime}$ y 6 $22^{\prime} 10^{\prime \prime}$ de latitud sur y los $79^{\circ} 13^{\prime} 15^{\prime \prime}$ y $81^{\circ} 19^{\prime} 35^{\prime \prime}$ de longitud oeste del Meridiano de Greenwich. Figura1.

Esta circunscripción colinda al oeste con el océano Pacífico y limita con los departamentos Lambayeque al sur, con Cajamarca al este y Tumbes por el noroeste, así como con territorio ecuatoriano por el noreste. Comprende una dilatada planicie en su mayor extensión Costa y una región montañosa menos extensa en la zona oriental del departamento Sierra. Desde la zona montañosa discurren los ríos Piura y Chira, que irrigan las excepcionales zonas cultivadas de la planicie costera, donde se extiende el desierto peruano y el bosque seco ecuatorial.

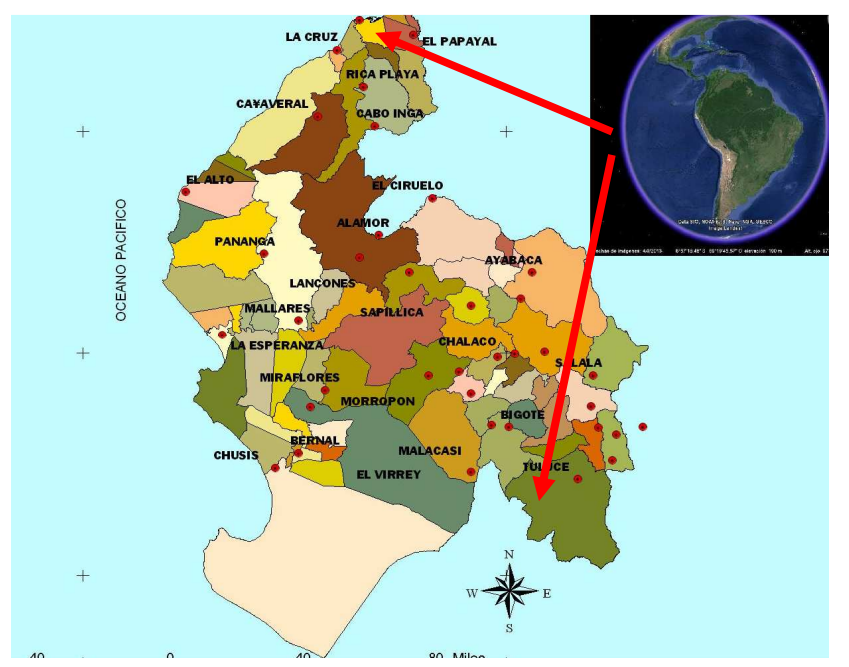

Figura 1. Ubicación del área de estudio

De acuerdo al diagrama bioclimático de Holdridge [20], presentan una temperatura media anual de $24^{\circ} \mathrm{C}$, un promedio de precipitación total anual que varía entre 81,0 y $260 \mathrm{~mm}$ incluido una estacionalidad marcada según el régimen de precipitaciones (con 9 meses secos y 3 meses de lluvias) presentando además un constante déficit hídrico.

\subsection{Estaciones de Monitoreo.}

Fueron seleccionadas seis localidades para el registro latitudinal permanente de los datos ubicados de radiación ultravioleta en la Costa y Sierra de la Región Piura-Perú, durante el verano del año 2008-2015. Su ubicación geográfica es descrita en la Tabla 1 y será útil en el proceso de interpolación.

Tabla 1. Localidades seleccionadas para el registro latitudinal de datos de radiación ultravioleta.

\begin{tabular}{lllll}
\hline Estación & Dpto & Longitud & Latitud & Altitud \\
\hline Miraflores & Piura & 80.6169 & 5.1669 & 30 \\
\hline Mallares & Piura & 80.7336 & 4.8503 & 45 \\
\hline Chulucanas & Piura & 80.1669 & 5.1003 & 95 \\
\hline Huancabamba & Piura & 79.46 & -5.24 & 1952 \\
\hline Ayabaca & Piura & 79.7169 & 4.6336 & 2700 \\
\hline Pacaypampa & Piura & 79.6627 & 4.9931 & 2054 \\
\hline
\end{tabular}

\subsection{Medición y Cálculo de la Radiación Ultravioleta.}

La determinación del índice según los niveles de Radiación Ultravioleta UV-B fueron medidos en la ciudad de Piura (distrito de Castilla lugar donde se ubican las oficinas del SENAMHI-Piura). Se trata del sensor IUV biometer modelo 501-A (Solar Light), SN 10734 (respuesta espectral 250/380 nm), periodo 20082011. Su ubicación geográfica se representa en la Tabla 2.

Tabla 2: Ubicación de la estación de monitoreo de radiación solar IUV de SENAMHI Piura

\begin{tabular}{llll}
\hline Localidad & Lat. & Long & $\begin{array}{l}\text { Altitud. } \\
\text { m.s.n.m }\end{array}$ \\
\hline Piura & 5011'18.58” $^{\circ 1}$ & 8037'02.49” & 29 \\
\hline
\end{tabular}


Ing. USBMed, Vol. 7, No. 1, Enero-Julio 2016

Para la determinación del índice de radiación ultravioleta, empleamos el espectro de acción eritemática inducido por radiación UV sobre la piel humana, de la CIE (Comisión Internationale de l'Eclairage). Parámetro adimensional que da cuenta de la radiación UV relevante para las personas, y viene definido por la Ecuación (6).

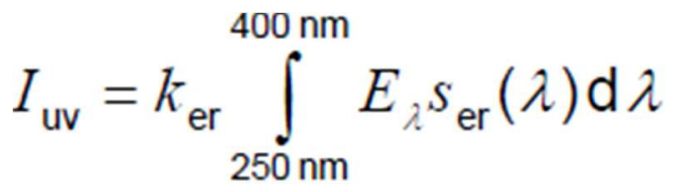

Donde I (I) representa a la irradiancia solar espectral ultravioleta expresada en $\mathrm{W} /\left(\mathrm{m}^{2} \cdot \mathrm{mm}\right)$ a la longitud de onda $\mathrm{I}, \mathrm{e}(\mathrm{I})$ es el espectro de acción eritemática desarrollado por la CIE y $\mathrm{ker}_{\text {e }}$ es una constante igual a 40 $\mathrm{m}^{2} / \mathrm{W}$.

La integral tiene como límite inferior $290 \mathrm{mn}$, por debajo del cual toda la radiación es absorbida antes de llegar a la superficie de la Tierra, y el límite superior (400 $\mathrm{nm}$ ) constituye la longitud de onda frontera entre el UV-A y el espectro visible.

Desde un aspecto cualitativo, hemos empleado la escala de valores del Índice UV, definida en una escala de 1 a 14+ según la Meteorological World Organization con un nivel de riesgo correspondiente a seis niveles (Mínimo, Bajo, Moderado, Alto, Muy Alto y Extremo). Clasificación importante tomando en consideración las recomendaciones efectuadas por las instituciones dermatológicas y que identificada según la Tabla 3.

Tabla. 3. Clasificación de niveles de radiación ultravioleta y niveles de Riesgo

\begin{tabular}{ll}
\hline Valor del Índice & Nivel de Riesgo \\
\hline Piura & Mínimo \\
\hline $1-2$ & Bajo \\
\hline $3-5$ & Moderado \\
\hline $6-8$ & Alto \\
\hline $9-11$ & Alto \\
\hline $12-14$ & Muy Alto \\
\hline+14 & Extremo \\
\hline
\end{tabular}

\section{Resultados}

\subsection{Variación horaria del IUV}

Los resultados de las mediciones reflejan la dependencia de los niveles de radiación ultravioleta respecto a la hora del día. Durante el transcurso de cada hora y a lo largo del día se observa variaciones del comportamiento de los niveles de radiación ultravioleta, debido al efecto de las nubes y/o a los cambios en la posición del sol. Los efectos que se derivan de la exposición a dicha radiación aumentan a medida que disminuye la masa de aire atravesada por la radiación solar y, por tanto, dependen de la hora solar. En el periodo observado, cinco niveles de radiación ultravioleta de acuerdo a la influencia horaria han sido identificados para la ciudad de Piura (mínimo, bajo, moderado, elevado y muy elevado).
La distribución horaria no es permanente en el tiempo iniciándose sus primeros e inocuos registros entre las 6:00 a 8:00 a.m, hora donde se registra una "mínima radiación" (Figura 3 ).

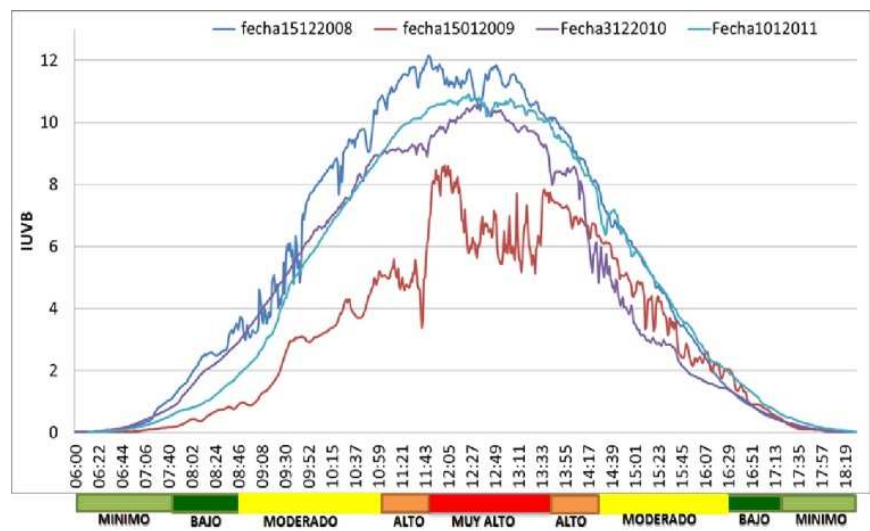

Figura 3. Distribución del IUV horario de 4 días observados dentro del periodo de observación

De acuerdo a la Figura 3, el aumento del IUV, de forma progresiva intensifica sus niveles entre las 11:00 a.m. a 14.30 horas (tiempo de mayor cuidado de sobre exposición solar que se debe tener sobre la piel). Los valores máximos de radiación ultravioleta se registran en torno al mediodía solar niveles "Alto" y "muy Alto". Este comportamiento se debe al hecho de que el ángulo cenital solar y por tanto, el camino que la radiación recorre a través de la atmósfera logran alcanzar la superficie donde sus valores son mínimos al iniciar la mañana y máximos al medio día.

La Figura 3, muestra la variación del índice UV influenciados por días con cielos claros (sin nubes) y bajo cielo nublado (condiciones meteorológicas propias debido a comportamiento local). Observamos que el día 15/12/2008, el IUV máximo registrado fue de 12.3 IUV a las 12:00 horas (nivel muy alto), mientras que el 15/01/2009 fue de 8,3 IUV (nivel moderado). De igual forma el día 31/12/2010 el máximo valor alcanzó un valor de 10.2 IUV (nivel alto) a las 12:37 horas, menor al registro el día 10/12/2011 donde el IUV fue de 10.3 IUV (nivel alto) a la misma hora que el registrado el 10/12/2011.

De forma general, transcurridas las 14:30 horas de la tarde, los niveles de radiación ultravioleta inician su progresivo descenso hasta las 18:00 horas donde la radiación es mínima (entre 1 a 2 IUV, Figura 3).

\subsection{Medición y Cálculo de la Radiación Ultravioleta}

Los resultados de las mediciones reflejan la dependencia de los niveles de radiación ultravioleta respecto a la hora del día. Durante el transcurso de cada hora y a lo largo del día se observa variaciones del comportamiento de los niveles de radiación ultravioleta, debido al efecto de las nubes y/o a los cambios en la posición del sol. Los efectos que se derivan de la exposición a dicha radiación aumentan a medida que 
disminuye la masa de aire atravesada por la radiación solar y, por tanto, dependen de la hora solar. En el periodo observado, cinco niveles de radiación ultravioleta de acuerdo a la influencia horaria han sido identificados para la ciudad de Piura (mínimo, bajo, moderado, elevado y muy elevado, Figura 4).

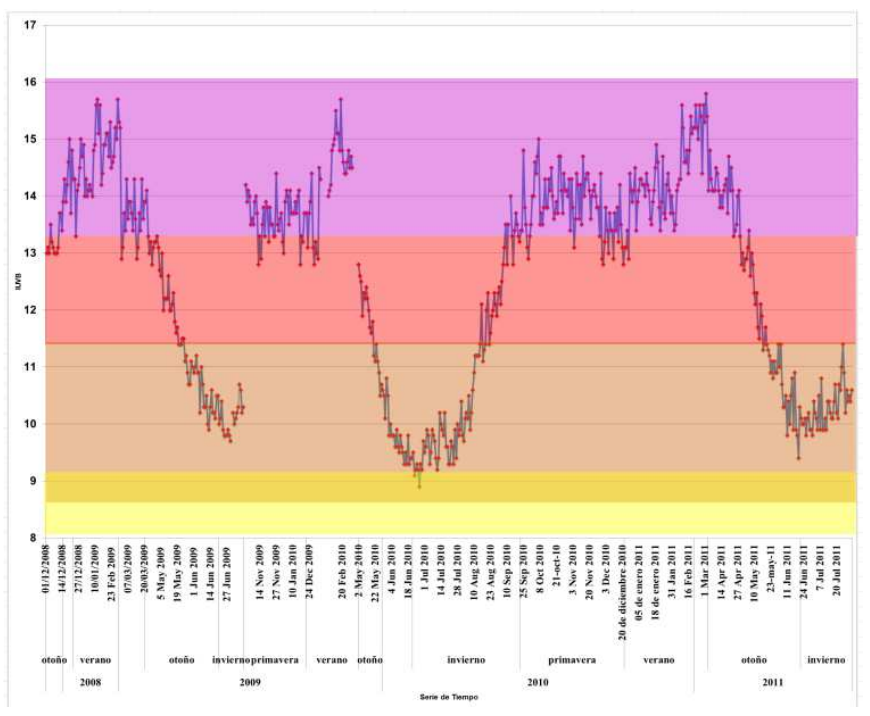

Figura 4. Distribución estacional del Índice de radiación ultravioleta para la ciudad de Piura

La menor incidencia de los niveles de radiación se produce a fines de otoño e inicios de invierno (dos últimas décadas de junio y primera década de julio). Es el caso del día 26 de junio del 2010 donde se registró el valor más bajo durante el periodo de observación (promedio de 8 IUV o nivel moderado en color amarillo).

Desde la segunda década de julio el índice IUV inicia su progresivo ascenso, situación coincidente con las estaciones de primavera y verano respectivamente. Desde el mes de noviembre, el riesgo de someterse a una exposición excesiva de la radiación, al mediodía solar se hace más frecuente hasta alcanzar niveles entre "muy altos" color rojo a "extremos" en color púrpura (Índice IUV > 14).

Es el caso de los valores registrados en verano entre febrero y la primera semana de marzo del 2009 donde los niveles de radiación ultravioleta superaron los 15 IUV (nivel extremo) en color purpura.

\subsection{Distribución Espacial}

La dinámica de la cobertura espacial del índice de radiación ultravioleta, desde los valores interpolados en las estaciones de observación, indica su inestabilidad en el tiempo (Figura 5).

En color verde oscuro se encuentran concentrados los mayores índices de radiación, con valores superiores a 14 IUV-B del cual inciden en mayor profundidad durante el mes de febrero concentrados sobre los distritos ubicados en la sierra de la región.

Su impacto, se va degradando a medida que descienden los rangos altitudinales hasta llegar a las partes más bajas identificadas en color verde claro y valores inferiores a 14 IUV-B.

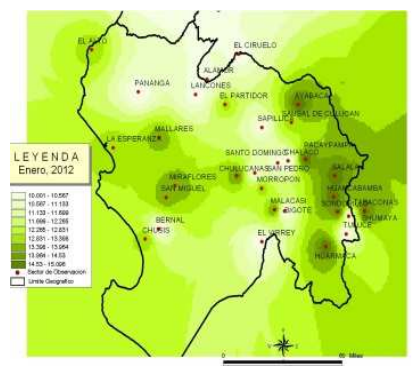

enero

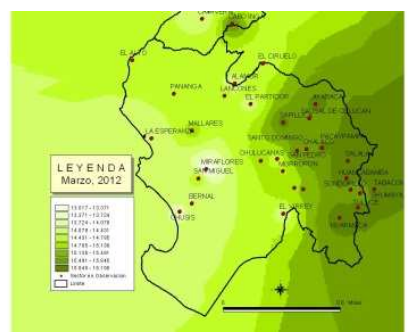

marzo

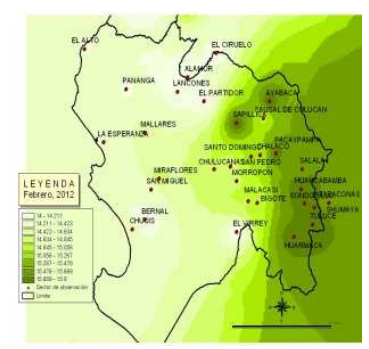

Febrero

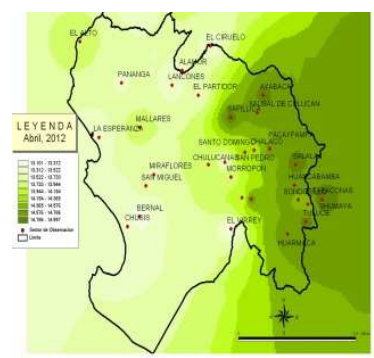

abril
Figura 5. Distribución espacial según niveles de radiación Ultravioleta IUV-B.

\section{Conclusiones}

Existe una influencia horaria en el comportamiento del índice de radiación ultravioleta siendo las horas donde se debe tener un mayor cuidado y protección en la piel entre las 11:00 a.m. a 14:30 p.m.

Del período observado, febrero es el mes que registra los mayores índices de radiación ultravioleta en costa y sierra.

Se corrobora la influencia directa de la altitud sobre los niveles de radiación ultravioleta siendo Ayabaca, la provincia que representa los valores más altos de niveles de radiación ultravioleta UVB de la región. Por ello al considerar que en el mes de febrero se desarrollan diversas actividades al aire libre dada la temporada de verano, es recomendable que estas se realicen a tempranas horas de la mañana o después de las cinco de la tarde. Una adecuada campaña educativa es la mejor herramienta para disfrutar inteligentemente de los beneficios del sol.

La estacionalidad es un factor determinante en el comportamiento del IUV durante el tiempo observado en el que se identifica a los meses entre junio y julio como los de menor registro descartándose durante este tiempo días con niveles extremos de radiación ultravioleta. 
Durante el verano entre los meses de febrero y marzo se registran días con niveles extremos de radiación ultravioleta.

Es recomendable la aplicación o uso de cremas protectoras o "bloqueadoras" (no son lo mismo que "bronceadoras") con un SPF por lo menos de 30, prendas protectoras como gorras o sombreros, lentes oscuros con foto protección.

\section{Referencias}

[1] C. Córdoba., Pérez, Aguirre., De Carcer, I.A., Jaque, F., Enriques, D., Carvalho, Fernando. "Comparison of total ozone measurements from a differential optical absorption filter instrument and a Dopson Spectrophotometer". En International Journal of Remote Sensing 18(16), pp. 3473-3478, 1997.

[2] Herman, Jay., Matthew R. Hudson, Rich, Mc Peters, Stolarski, Richard, Ahmad, Ziauddin Gu, X. Y., Taylor, S and Wellemeyer, C.A. "New Self-Calibration Method Applied to TOMS and SBUV Backscattered Ultraviolet Data to Determine Long-Term Global Ozone Change", En J. Geophys. Res., Vol. 96, pp. 75317545.1991

[3] Herman, Jay. R., Rich. McPeters and DLarko. "Ozone Depletion at Northern and Southern Latitudes Derived from January 1979 to November 1991, Total Ozone Mapping Spectrometer", En J. Geophys. Res., Vol. 98, pp. 12783.1993.

[4] D. Bojkov Rumen., Lane. Bishop and Vitali.E. Fioletov;.."Total Ozone Trends from Quality-Controlled Ground Base Data (19641994)", En J. Geophys. Res., Vol. 100, pp. 25867-25876, 1995

[5] Tevini, Manfred.; "UV-B Radiation and Ozone Depletion.Effects on Humans, Animals, Plants, Microorganism and Materials". Chapter 4 in UV-B radiation and ozone depletion: ed. pp. 95-123. Ann Arbor: Lewis Publishers, 1993.

[6] Grant, Richard. H., Gordon. M. Heisler, and Guan. Gao. Clear sky radiance distributions in ultraviolet wavelength bands. J. Theor. and Applied Climatol. Vol. 56, pp.123-135, 1997.

[7] Norris, P G, Hawk J L, "Polymorphic light eruption. Photodermatol Photoimmunol”. En Photomed. 7(5) pp. 186-191, 1990.

[8] Clydesdale Gavin, J, Dandie Geoffrey W, Muller H.Konrad, "Ultraviolet light induced injury: immunological and inflammatory effects". En Immunol Cell Biol. 79, pp. 547-568, 2001.
[9] Fisher, Gary.J, "The pathophysiology of photoaging of the skin". En Cutis. 75: pp. 5-8, 2005[7]Norris, P G, Hawk J L, "Polymorphic light eruption. Photodermatol Photoimmunol”. En Photomed. 7(5) pp. 186-191, 1990..

[10] Claerhout, Sofie, Van Laethem. Aethema A, Agostinis Patrizia, Garmyn Marian, "Pathways involved in sunburn cell formation: Deregulation in skin cancer". En Photochem. Photobiol. Sci. 5: pp.199-207, 2006...

[11] Gallagher, Richard. P, Lee T. K. Adverse effects of ultraviolet radiation: a brief review. Prog. Biophys. Mol. Biol. 92: pp.119-131, 2006.

[12] De Gruijl. Frank R, Photo carcinogenesis: "UVA vs. UVB radiation". En Skin Pharmacol Appl Skin Physiol 15: pp. 316-320, 2002.

[13] González, Pumariega, Maribel, Vernhes, Tamayo. Marioly. Sánchez, Lamàr. Ángel, "La Radiación Ultravioleta. Su Efecto Dañino y Consecuencias Para La Salud Humana". En Theoria Vol. 18 (2), pp. 69-80, 2009

[14] Tortora, Gerard, y Gravoski, Sandra Reynols. Principios de Anatomía y Fisiología. 11 Edición. Editorial Médica Panamericana, pp. 550, 1993.

[15] Sánchez, Saldaña, L. Radiación ultravioleta y cáncer a la piel. Dermatología Peruana, Vol 19(4) 305, 2009.

[16] Valverde-López, Jenny., Querevalú-Eche, Félix., Tincopa-Wong, Oscar. "Photodermatosis-Clinical and Epidemiologic Traits in a General Hospital”. En Dermatol Perú 15(2), pp.113-120, 2005.

[17] Moncada, Jiménez. José: La Radiación Ultravioleta y la Piel del Deportista. En Redalic. http://www.redalyc.org/articulo.oa?id=44027211, 1993.. Última consulta 25 julio de 2013.

[18] OMS. Las radiaciones ultravioleta y la salud humana. Nota descriptiva $\quad N^{\circ} \quad 305 . . \quad$ Disponible en http://www.who.int/mediacentre/factsheets/fs305/es, 2005. Última consulta, 25 de Julio de 2013.

[19] INEI. Censos Nacionales XI de Población y VI de Vivienda, 21 de octubre del 2007, Perú: Resultados Definitivos, Tomo I. Lima septiembre del 2008.

[20] L. Holdridge,..,Ransselaer.. «Life Zone Ecology». Tropical Science Center. San José, Costa Rica. (Traducción del inglés por Humberto Jiménez Saa: «Ecología Basada en Zonas de Vida», 1a. ed. San José, Costa Rica: IICA, pp. 149. 1982. Disponible en http://cct.or.cr/publicaciones/Life-Zone-Ecology.pdf. Última consulta 1 Agosto de 2013. 